H I G H L I G H T S

\section{Silent partner speaks out}

The cytokine milieu is crucial for determining the outcome of an immune response after T-cell-B-cell interaction, but most studies have focused on the cytokines produced by T cells. Now, it seems that B cells might have a more active role in determining the fate of immune responses than previously thought. New research in The Journal of Immunology has shown that B cells produce a dynamic profile of cytokines depending on the strength, timing and nature of stimulation they receive.

The authors set up an in vitro system for B-cell stimulation using freshly isolated human B cells, B-cell receptor (BCR)-specific antibodies to mimic crosslinking with antigen, and mouse fibroblasts stably transfected with CD40L to mimic T-cell help. In vivo experiments have shown that BCR engagement alone does not induce B-cell proliferation, whereas CD40 engagement alone induces proliferation followed by apoptosis, and engagement of both BCR and CD40 results in sustained proliferation. By altering the concentration of antibody and the ratio of $\mathrm{CD} 40 \mathrm{~L}^{+}$fibroblasts to $\mathrm{B}$ cells, they were able to mimic these three situations in vitro, creating a model that effectively reproduces the physiological situation.

In this system, B cells stimulated with BCR crosslinking alone (mimicking the absence of T-cell help) did not produce significant levels of

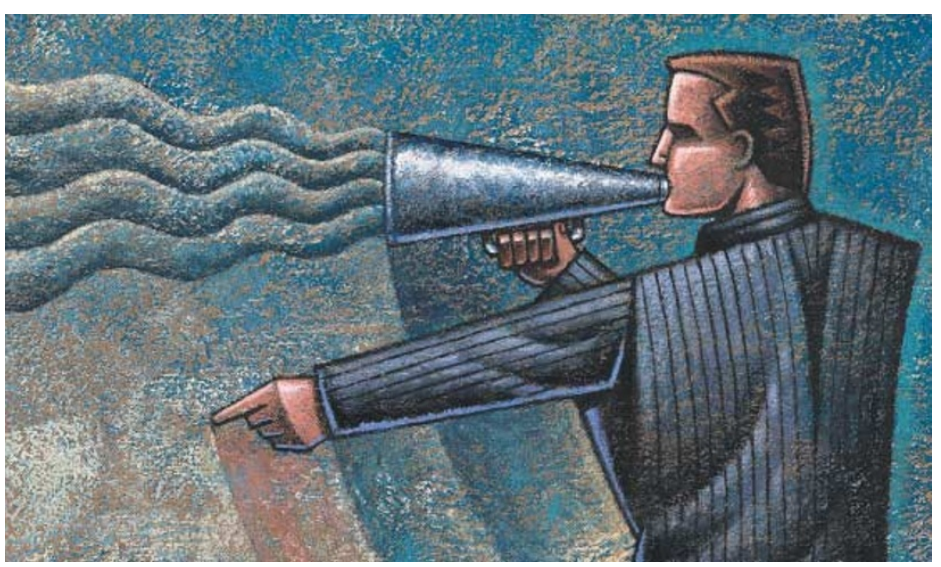

cytokines. However, when BCR crosslinking was combined with stimulation through CD40, the B cells produced high levels of tumour necrosis factor (TNF), lymphotoxin and interleukin-6 (IL-6) in a dosedependent manner, which would promote an ongoing immune response. Staggered dual activation (24 hours of BCR crosslinking before addition of $\mathrm{CD}_{40 \mathrm{~L}^{+}}$fibroblasts) amplified this cytokine response. This mimics the sequence of interactions in vivo, where a B cell typically encounters antigen in the B-cell zone of a lymph node before migrating to $\mathrm{T}$-cell areas to receive help from CD40L ${ }^{+} \mathrm{T}$ cells.

By contrast, stimulation through CD40 alone resulted in production of the immunosuppressive cytokine IL-10 by B cells, in the absence of TNF and lymphotoxin. CD40 is expressed constitutively by B cells, so the production of IL-10 could help to ensure that bystander T-cell help does not result in inappropriate B-cell responses in the absence of cognate antigen.

This study has shown that B-cell cytokines can both amplify desirable immune responses and inhibit inappropriate responses. The moral of the story is that T cells don't always wear the trousers!

Kirsty Minton

(2) References and links ORIGINAL RESEARCH PAPER Duddy, M. E. et al. Distinct profiles of human $B$ cell effector cytokines: a role in immune regulation? J. Immunol. $\mathbf{1 7 2}$ 3422-3427 (2004)

\section{IN BRIEF}

INNATE IMMUNITY

\section{A Toll-like receptor that prevents infection by} uropathogenic bacteria.

Zhang, D. et al. Science 303, 1522-1526 (2004)

In this study, Zhang et al. identified a novel Toll-like receptor, termed TLR11, which seems to sense uropathogenic bacteria and initiate immune responses that lead to their clearance. TLR11 was discovered in a mouse liver expressed-sequence tag database during a search for sequences that have homology with the Toll/interleukin-1 receptor domain. Further experiments showed abundant expression of TLR11 by both kidney and bladder epithelial cells. TLR11-deficient mice were subsequently found to be highly susceptible to kidney infections after intra-urethral challenge with uropathogenic Escherichia coli. The authors speculate that humans are susceptible to urinary-tract infections because they have a truncated form of TLR11, which might be functionally inactive.

\section{CELL MIGRATION}

Locomotion of monocytes on endothelium is a critical step during extravasation.

Schenkel, A. R. et al. Nature Immunol. 5, 393-400 (2004)

The current paradigm of leukocyte extravasation states that cells undergo a sequential series of steps when migrating from the blood into tissues - tethering, rolling, adhesion and diapedesis. So, how do leukocytes that adhere to the endothelium locate and move towards the endothelial-cell junctions where diapedesis occurs? In this paper, the interaction of monocyte $\beta_{2}$ integrins with their respective endothelial ligands, which is known to mediate adhesion, was also shown to have a distinct role in the subsequent 'locomotion' to cellular junctions. Blockade of either $\beta_{2}$ integrins or their endothelial ligands caused monocytes to spin and fail to position themselves at cellular junctions, thereby defining a new step in the regulation of leukocyte diapedesis.

\section{TUMOUR IMMUNOLOGY}

Innate immune surveillance of spontaneous B cell lymphomas by natural killer cells and $\gamma \delta$ T cells.

Street, S. E. A. et al. J. Exp. Med. 199, 879-884 (2004)

The extent to which innate immune cells contribute to immune surveillance against tumours is still controversial. In this paper, Street et al. studied the mechanism by which wild-type mice reject transplants of MHC class-I-deficient B-cell lymphomas. These tumour cells were found to be rejected by mice lacking various immune-cell populations - except those depleted of natural killer (NK) cells or NK cells and $\gamma \delta$ T cells. This tumour-specific response was shown to be perforin dependent, because perforin-deficient mice succumbed to spontaneous development of B-cell lymphomas. Therefore, this study indicates a crucial role for innate immune cells in the surveillance of B-cell lymphomas. The mechanism by which NK cells and $\gamma \delta$ T cells are stimulated by MHC class-I-deficient tumour cells remains unclear. 